\title{
ARTICLE OPEN Regulation of sister chromatid cohesion by nuclear PD-L1
}

\author{
Jia Yu $\mathbb{D}^{1}$, Bo Qin ${ }^{1,2}$, Ann M. Moyer $\mathbb{D}^{3}$, Somaira Nowsheen $\mathbb{D D}^{4}$, Xinyi Tu${ }^{2}$, Haidong Dong ${ }^{5}$, Judy C. Boughey ${ }^{6}$, Matthew P. Goetz ${ }^{1,2}$, \\ Richard Weinshilboum ${ }^{1}$, Zhenkun Lou iD $^{2}$ and Liewei Wang ${ }^{1}$
}

Programmed death ligand-1 (PD-L1 or B7-H1) is well known for its role in immune checkpoint regulation, but its function inside the tumor cells has rarely been explored. Here we report that nuclear PD-L1 is important for cancer cell sister chromatid cohesion. We found that depletion of PD-L1 suppresses cancer cell proliferation, colony formation in vitro, and tumor growth in vivo in immunedeficient NSG mice independent of its role in immune checkpoint. Specifically, PD-L1 functions as a subunit of the cohesin complex, and its deficiency leads to formation of multinucleated cells and causes a defect in sister chromatid cohesion. Mechanistically, PDL1 compensates for the loss of Sororin, whose expression is suppressed in cancer cells overexpressing PD-L1. PD-L1 competes with Wing Apart-Like (WAPL) for binding to PDS5B, and secures proper sister chromatid cohesion and segregation. Our findings suggest an important role for nuclear PD-L1 in cancer cells independent of its function in immune checkpoint.

Cell Research (2020) 30:590-601; https://doi.org/10.1038/s41422-020-0315-8

\section{INTRODUCTION}

Immune checkpoints are cell surface receptors that are expressed in immune cells. They modulate the amplitude and quality of the adaptive and innate effectors, thus maintaining immune homeostasis and preventing autoimmunity. ${ }^{1}$ Well-studied immune checkpoint proteins include cytotoxic T-lymphocyte protein 4 (CTLA4) and programmed cell death protein 1 (PDCD1; also known as PD1). CTLA4 pathways restrict T cell activity at the early stage of immune response, whereas PD1 signaling plays roles in the later stage of immune response, protecting surrounding tissues at sites of chronic inflammation from damage. ${ }^{2}$ Both immune checkpoints are utilized by cancer cells to evade immune surveillance. PD1, also known as PDCD1 or CD279, is a membrane protein and expressed on T cells and pro-B cells. ${ }^{3-5}$ Its ligand, PD$\mathrm{L} 1$, also referred to as $\mathrm{B} 7-\mathrm{H} 1$ or $\mathrm{CD} 274$, is a type 1 transmembrane protein that binds to PD1 and negatively regulates $\mathrm{T}$ cell function and survival. ${ }^{6}$ The expression of PD-L1 in the tumor microenvironment protects cancer from immune-mediated rejection. ${ }^{7}$ PD-L1 is up-regulated in a variety of human carcinomas, including breast cancer, ovarian cancer, colon cancer, melanoma and lung cancer. ${ }^{7,8}$ PD-L1 assists tumor cells evade the host immune system. Consequently, several checkpoint inhibitors, such as antiPD-L1 monoclonal antibody, have been developed to block this inhibitory pathway and reactivate T-cell activity against cancer cells. ${ }^{6,9}$ High levels of PD-1 expression are detected in circulating and tumor-infiltrating lymphocytes in different cancer tissues. ${ }^{10-13}$ These antibodies function by blocking tumor PD-L1-PD-1 interaction that preserves the PD-1 expressing anti-tumor T cell function, so that they can attack tumor cells expressing PD-L1.

Due to the huge success of anti-PD-L1 and anti-PD-1 therapy, much effort has been put into studying the interaction between the tumor PD-L1 and T cell PD1 in cancer immunotherapy. Recently, only a few studies have explored the functions of PD-L1 inside tumor cells. These studies suggest that tumor PD-L1 suppresses tumor apoptosis, modulates chemo-resistance through MAPK/ERK activation, controls tumor glucose metabolism and regulates autophagy in ovarian cancer and melanoma. ${ }^{14-16}$ Interestingly, nuclear staining of PD-L1 is detected in cancer tissues. ${ }^{17}$ However, the function of nuclear PD-L1 in cancer cells is rarely explored.

PD-L1 is overexpressed in $\sim 20 \%$ of triple negative breast cancers (TNBCs), ${ }^{18}$ a subtype of breast cancer with a very poor prognosis. Recent phase I studies using antibodies that target PDL1 (atezolizumab) in women with metastatic TNBC have demonstrated that a minority of women $(<10 \%)$ exhibit tumor response. ${ }^{19,20}$ These studies prompted us to use TNBC as one of the models to study PD-L1 function in tumor.

Cohesin is a highly conserved chromosome-associated multisubunit protein complex in eukaryotes. It is composed of four core subunits-SMC1, SMC3, SCC1 (RAD21), and either SA1 (STAG1) or SA2 (STAG2) and three regulatory subunits-PDS5B, WAPL and Sororin. ${ }^{21-28}$ The cohesin core subunits form a ring-shaped structure. SMC1 and SMC3 dimerize with hinge domain and their other ends are bridged by SCC1. SA $1 / 2$ and PDS5B associate with SCC1. Through PDS5B, the sub-stoichiometric regulator WAPL and Sororin control the ring open and close status, ${ }^{29,30}$ and this structure topologically encircles chromatin. Cohesin is loaded onto chromatin in early G1 phase and established during DNA replication in $S$ phase. ${ }^{22,31}$

Here we report a novel role of nuclear PD-L1 in regulation of the cohesin complex. Our findings show that PD-L1, compensating for the loss of Sororin, competes with WAPL binding to PDS5B and

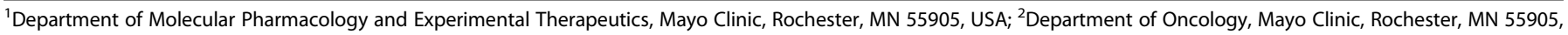

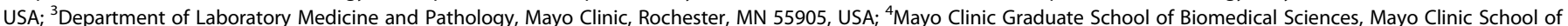

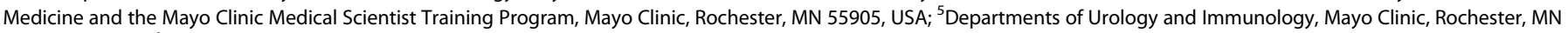
55905, USA and ${ }^{6}$ Department of Surgery, Mayo Clinic, Rochester, MN 55905, USA

Correspondence: Zhenkun Lou (Lou.Zhenkun@mayo.edu) or Liewei Wang (Wang.Liewei@mayo.edu)

These authors contributed equally: Jia Yu, Bo Qin.

Received: 2 July 2019 Accepted: 31 March 2020

Published online: 29 April 2020 


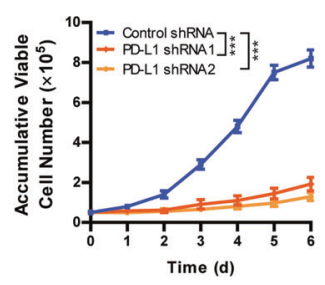

e

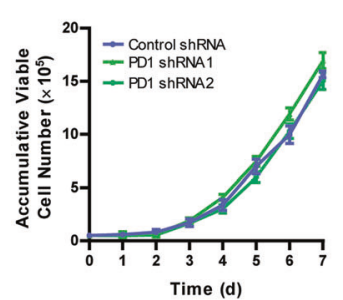

i

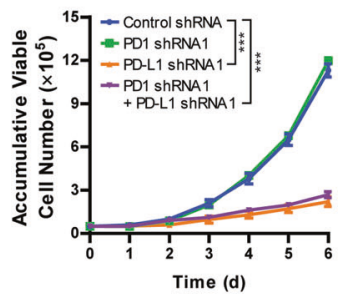

b
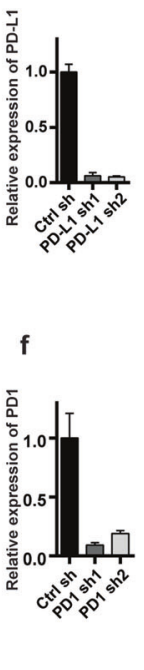

j

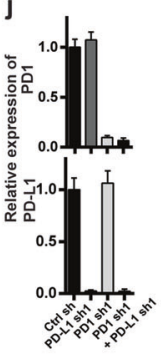

c

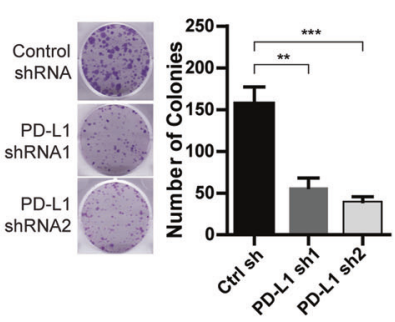

g

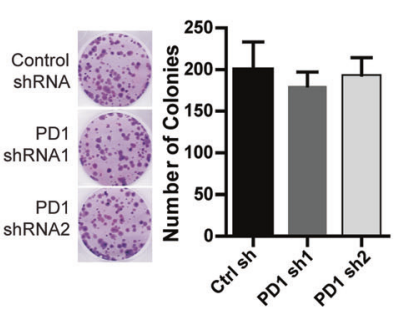

k

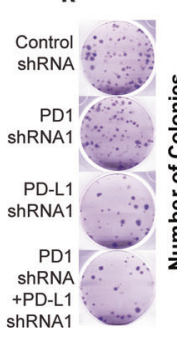

d
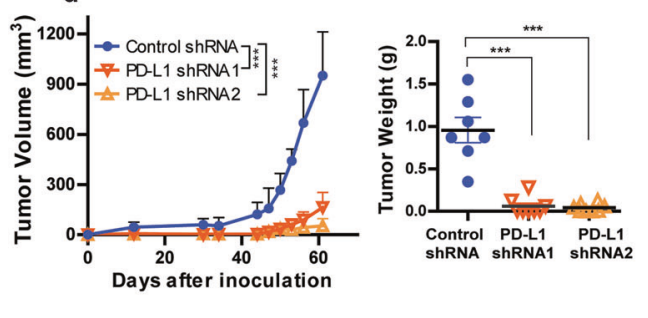

h
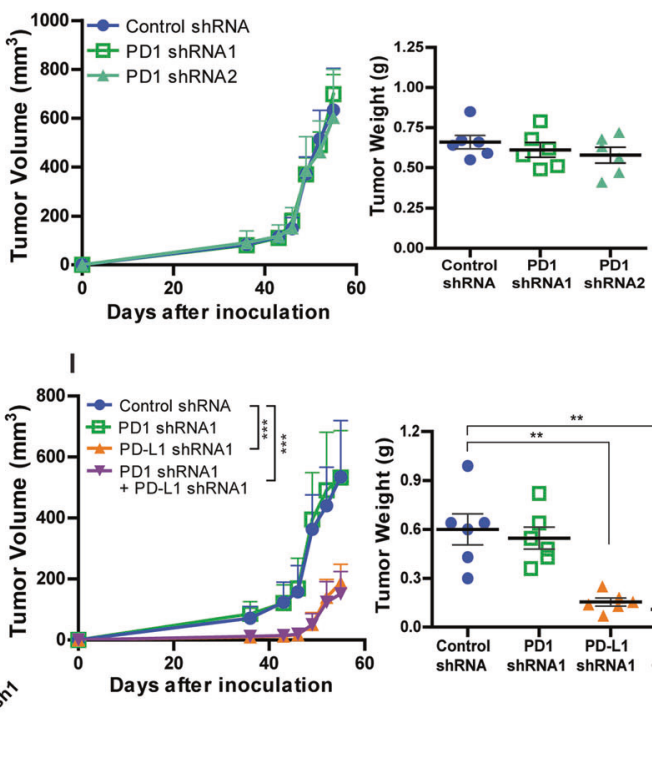

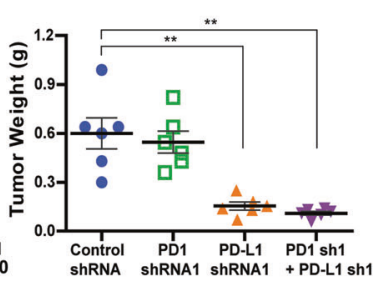

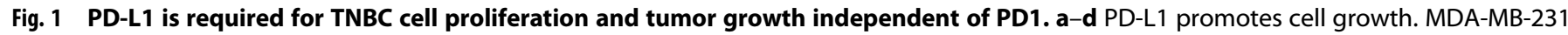

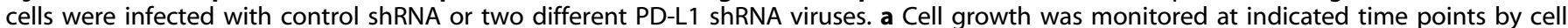
counting. b PD-L1 knockdown efficiency was determined by qRT-PCR. c Colony formation assays were performed. $\mathbf{d}$ In vivo tumor growth in NSG mice was assessed and tumor weights were measured when experiments were terminated. e-h PD1 is dispensable for cell growth. e MDA-MB-231 cells expressing control shRNA or two different PD1 shRNAs were monitored for cell proliferation at indicated time points by cell counting. PD1 knockdown efficiency (f), colony formation (g), and tumor growth in NSG mice (h) were determined, respectively. i-I PD-L1mediated cell proliferation is independent of PD1. i MDA-MB-231 cells expressing control shRNA, PD-L1 shRNA, PD1 shRNA, or a combination of PD-L1 shRNA and PD1 shRNA were monitored for cell growth. Knockdown efficiency (j) and colony formation (k) were independently replicated three times with similar results. I Tumor growth at different time points was determined and tumor weights were measured at the time when the experiments were terminated $(n=6-7)$. Data are presented as means \pm SEM of $n=3$ independent experiments. Student's $t$-test was used for the comparisons for (c, d) (right panel), (g, h) (right panel), (k, I) (right panel), and 2-way ANOVA was used for (a, d) (left panel), (e, h) (left panel), (i, I) (left panel). ${ }^{* *} P<0.01,{ }^{* *} P<0.001$.

regulates cohesin complex status and genomic stability in cancer cells. PD-L1 deficiency suppresses tumor growth in a PD-1 independent manner. Moreover, $P d-11$ knockout mice do not display cohesion defect, suggesting a unique role of PD-L1 in cancer cells.

\section{RESULTS}

PD-L1 is required for TNBC cell proliferation and tumor growth independent of PD1

We first suppressed PD-L1 expression in two TNBC cell lines that highly express PD-L1 to evaluate its effect on cellular phenotypes (Fig. 1a-d; Supplementary information, Fig. S1a, b). Interestingly, depletion of PD-L1 with two different shRNAs dramatically suppressed MDA-MB-231 cell proliferation and colony formation (Fig. 1a-c). To confirm this result, we also generated inducible $P D$ $L 1$ knockout cell lines. Knocking out $P D-L 1$ also greatly reduced colony formation in MDA-MB-231 cells (Supplementary information, Fig. S1a). A similar phenotype was observed in a second TNBC cell line, BT549 cells (Supplementary information, Fig. S1b). Based on expression of estrogen receptor (ER), progesterone receptor (PR), and human epidermal growth factor receptor 2 (HER2), breast cancer can be classified into three subtypes, including ER-positive breast cancer, HER2-positive breast cancer, and triple negative breast cancer (TNBC). Compared to TNBC cells, ER-positive or HER2-positive breast cancer cells, including MCF7, ZR-75-1, and BT474 cells, express very low levels of PD-L1. To test the effect of PD-L1 knockdown in both subtypes in addition to TNBC, we also transduced PD-L1 shRNA lentivirus into cell lines with various receptor status (Supplementary information, Fig. S1c). Interestingly, PD-L1 knockdown did not affect proliferation of these cells (Supplementary information, Fig. S1d-f), suggesting impaired cell survival is specific to cells that highly express PD-L1. PD-L1 has also been reported to be overexpressed in many different cancer types, including prostate, colon, melanoma, and ovarian cancers. To test whether our observation that PD-L1 is required for cell proliferation is generalizable to other cancers, we assessed PD-L1-mediated proliferation in cancer cell lines from different tissue origins, including lung, colon, and prostate. As expected, PD-L1 expression varied among cell lines, with several cell lines showing high PD-L1 expression (Supplementary information, Fig. S1g). Depletion of PD-L1 in these cells significantly suppressed colony formation (Supplementary information, Fig. S1h), suggesting that PD-L1 is important for proliferation in cancer cells that highly express PD-L1. 
a

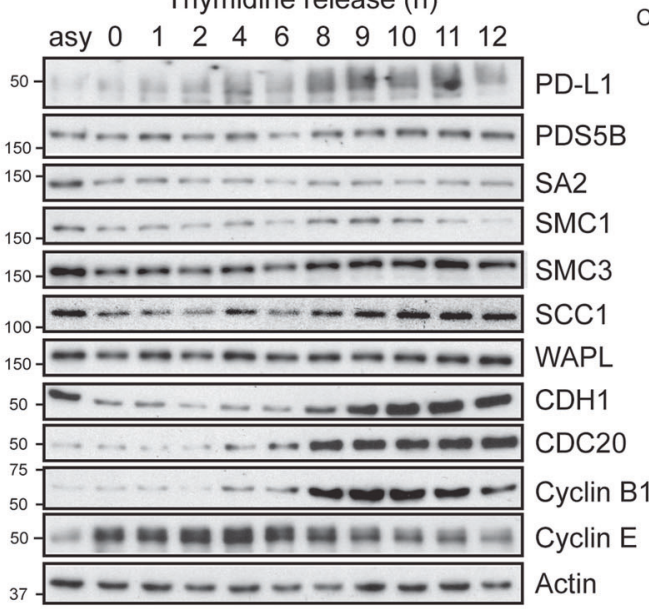

d Cancer Type $\frac{\text { Prostate Colon Lung }}{\text { 30 }^{0}}$ Breast

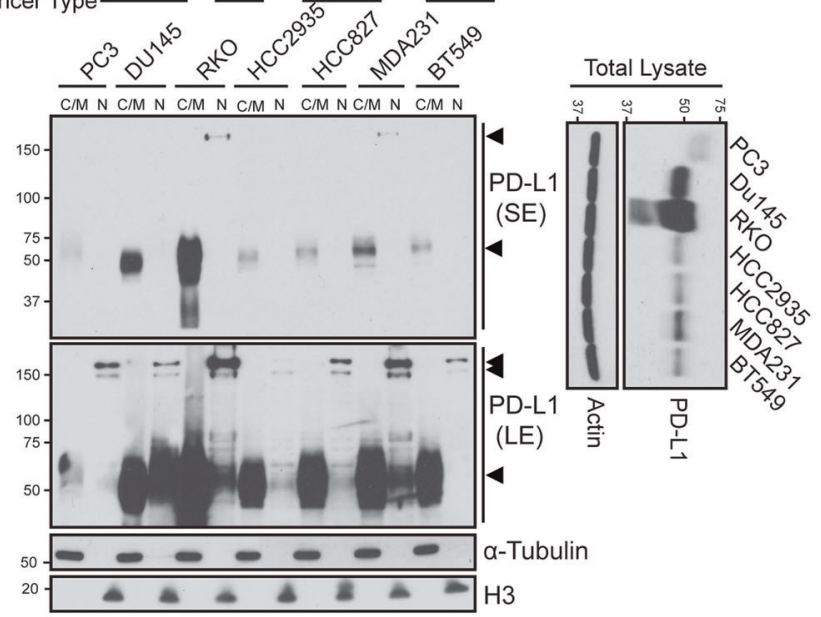

b

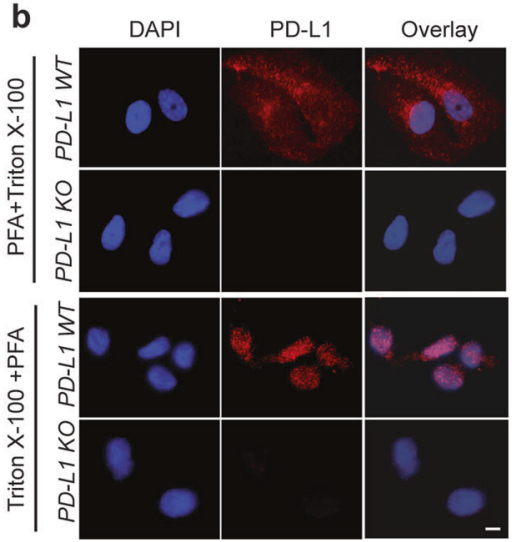

h

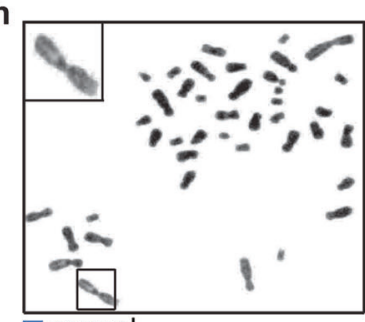

normal
C

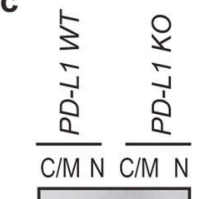

f Control
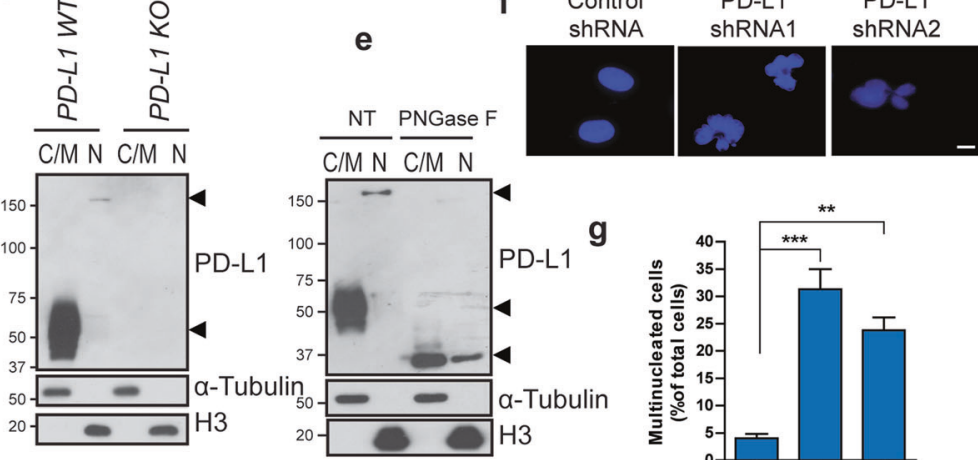

NT PNGase F $\overline{\mathrm{C} / \mathrm{M} \mathrm{N}} \overline{\mathrm{C} / \mathrm{M} \mathrm{N}}$
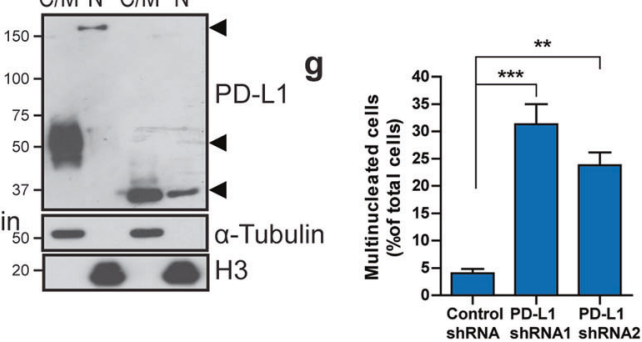

i

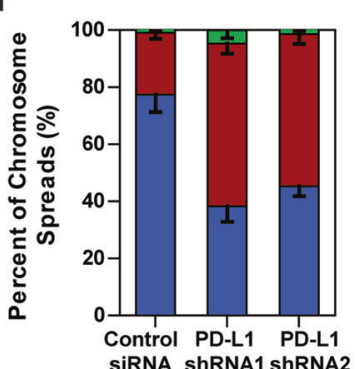

partial loss

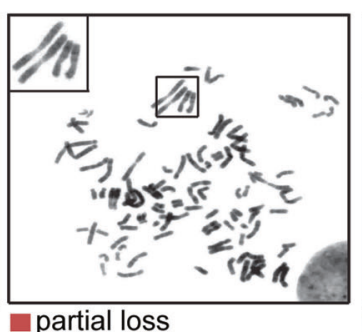

hPD-L1

shscC1

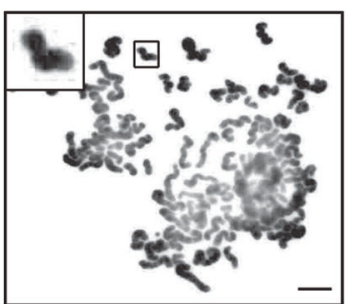

total loss SIRNA ShRNA1 shRNA2

$$
\text { j }
$$

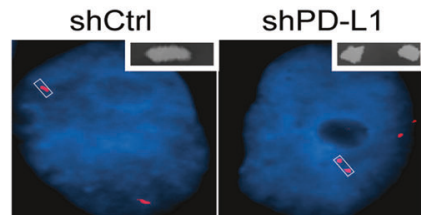
shSororin
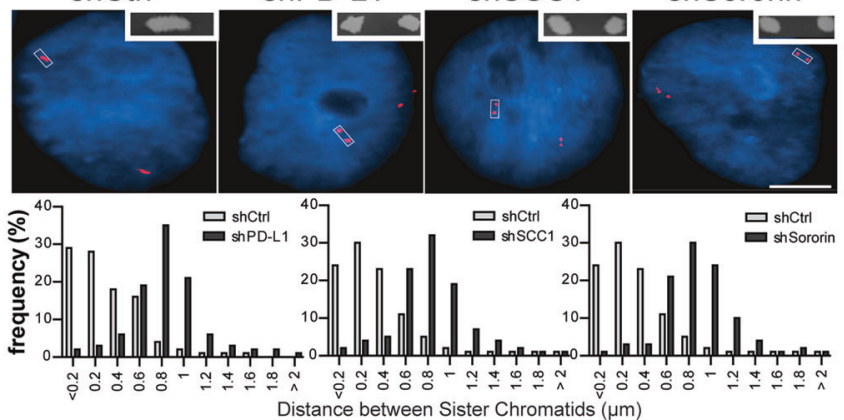

Distance between Sister Chromatids $(\mu \mathrm{m})$

To investigate whether loss of PD-L1 affects tumor growth in vivo, we injected MDA-MB-231 cells stably expressing control shRNA or two different PD-L1 shRNAs into NSG mice. These mice lack both innate and adaptive immunity, thereby being a perfect model to exclude PD-L1's immunosuppression effect. ${ }^{32}$ We found that depletion of PD-L1 in MDA-MB-231 cells greatly impeded tumor growth (Fig. 1d). These results indicate that PD-L1 contributes to TNBC cell proliferation and tumor growth.

PD1, the receptor for PD-L1, is widely expressed in many different cancers. ${ }^{32}$ Thus, we examined the expression of PD1 in 
Fig. 2 PD-L1 is a cell cycle dependent protein and regulates sister chromatid cohesion in TNBC. a PD-L1 level fluctuates during the cell cycle. Target proteins were detected by Western blot in MDA-MB-231 cells at indicated time points after double thymidine block. b Immunostaining of PD-L1 in interphase cells. Wildtype (WT) or PD-L1 knockout (KO) MDA-MB-231 cells were permeabilized and fixed using the indicated methods and cells were then stained with anti-PD-L1 antibody. PFA + Triton X-100: cells were fixed with 3\% paraformaldehyde followed by $0.5 \%$ Triton X-100. Triton X-100 + PFA: cells were treated with $0.5 \%$ Triton X-100 first to remove most cytoplasmic/membrane proteins, and followed by fixation with $3 \%$ paraformaldehyde. Nucleus was stained with DAPI. Scale bar: $10 \mu \mathrm{m}$. c Detection of PD-L1 in different fractions of control and PD-L1 knockout MDA-MB-231 cells. Cytoplasmic/membrane (C/M) and nuclear (N) fraction. d Cytoplasmic/ membrane $(C / M)$ and nuclear $(\mathrm{N})$ fractions of PD-L1 from different cancer cell lines. SE short exposure; LE long exposure. e Cytoplasmic/ membrane $(C / M)$ and nuclear $(N)$ fractions of MDA-MB-231 cells with or without PNGase $F$ treatment. Arrows indicate PD-L1 bands. $\mathbf{f}, \mathbf{g}$ Loss of PD-L1 promotes multinucleation. $\mathbf{f}$ Representative nuclear staining by DAPI in MDA-MB-231 cells three days post-infection with control shRNA virus or PD-L1 shRNA virus. Scale bar: $10 \mu \mathrm{m}$. g Quantification of multinucleated cells was performed. Data are presented as means \pm SD, and were independently replicated three times. Statistical significance was calculated using Student's $t$ test. ${ }^{* *} P<0.01,{ }^{* * *} P<0.001$. h, i PD-L1 is required for sister chromatid cohesion. $\mathbf{h}$ Representative metaphase spreads showing MDA-MB-231 cells with normal, partial loss and total loss of sister chromatid cohesion. Scale bar: $5 \mu \mathrm{m}$. i Quantification of different sister chromatid status in control and PD-L1 knockdown cells. Data are presented as means \pm SD, independently replicated three times with similar results. $\mathbf{j}$ FISH assays were performed in MDA-MB-231 cells expressing control shRNA, PD-L1 shRNA, SCC1 shRNA or Sororin shRNA. myb gene probe was used and the distance between paired FISH signals was measured and quantified. Bar: $5 \mu \mathrm{m}$. k Western blots showing the knockdown efficiency for the experiments in $\mathbf{j}$.

different subtypes of breast cancer. Distinct from the PD-L1 expression profile, which is overexpressed in TNBC (when compared to hormonal receptor positive or HER2 + breast cancer subtypes), PD1 levels varied little among the cell lines across different subtypes (Supplementary information, Fig. S1c). Kleffel et al have reported that in human melanoma cells, overexpression of PD1 enhances tumor proliferation. ${ }^{32}$ Therefore, we explored whether PD1 might regulate cell growth and tumor progression in TNBC. We depleted PD1 in MDA-MB-231 cells using two different shRNAs, but did not observe any significant changes in cell proliferation or colony formation (Fig. 1e-g). Similar results were obtained in BT549 cells (Supplementary information, Fig. S1i). To further confirm this finding, we injected MDA-MB-231 cells stably expressing control shRNA or PD1 shRNA into immunodeficient NSG mice. Consistent with the in vitro results, depletion of PD1 did not affect tumor growth in mice (Fig. 1h). These results suggest that in contrast to PD-L1, tumor cell expression of PD1 does not drive TNBC cell growth either in vitro or in vivo.

It has been reported that tumor intrinsic PD1 can interact with PD-L1 and enhance proliferation of melanoma cells by activating the mTOR pathway. ${ }^{32}$ To test whether the interaction between PDL1 and PD1 might affect TNBC cell growth, we treated MDA-MB231 and BT549 cells with increasing concentrations of anti-PD-L1 antibody that blocked the interaction between PD-L1 and PD1, and monitored cell growth. Interestingly, no inhibition of cell proliferation was observed when the cells were treated with an anti-PD-L1 antibody (Supplementary information, Fig. S1j, k). Next, we depleted PD-L1 and PD1 either separately or together in MDMB-231 cells (Fig. 1i-k). Consistently, knockdown of PD-L1, but not PD1, greatly suppressed cell proliferation and colony formation. Furthermore, knocking down PD1 in PD-L1 depleted MDA-MB-231 cells did not further affect either cell proliferation or colony formation (Fig. 1i-k), suggesting that the regulation of cell growth is independent of the interaction between PD-L1 and PD1 in these cells. To further confirm these results, we injected MDA-MB-231 cells stably expressing control shRNA, PD-L1 shRNA, PD1 shRNA, or PD1 shRNA plus PD-L1 shRNA into NSG mice, and monitored tumor growth. No significant difference in tumor growth was observed between the PD-L1 depleted tumor group and the PDL1 and PD1 double knockdown group (Fig. 1I). Taken together, these results indicate that PD-L1 contributes to TNBC cell proliferation and tumor growth in a PD1 independent manner.

PD-L1 is a cell cycle dependent protein and regulates sister chromatid cohesion in TNBC

To better understand the role of PD-L1 in TNBC cell proliferation, we examined the protein levels of PD-L1 during different cell cycle phases. Unexpectedly, we found that PD-L1 protein accumulates in the G2/M phase and decreases in G1 (Fig. 2a). These results suggest that PD-L1 expression in TNBC is cell cycle dependent. This observation is consistent with a recent report by Zhang et al which showed similar cell cycle dependent changes in PD-L1. ${ }^{33}$ Detection of PD-L1 signals in the nucleus by immunofluorescence labeling after permeabilization with Triton-X-100 suggested that PD-L1 protein associates with DNA (Fig. 2b). To further confirm the nuclear localization of PD-L1, we performed fractionation assays and detected PD-L1 in the nuclear fraction (Fig. 2c). Surprisingly, we found that nuclear PD-L1 was super-shifted (over $150 \mathrm{kDa}$ ) compared to the cytosolic/membrane fraction by Western blot, and this band was absent in $P D-L 1$ knockout cells (Fig. $2 \mathrm{C}$ ). The same super-shift bands for nuclear PD-L1 were observed in multiple cancer cells of different origins (Fig. 2d). Treatment of the therapeutic PD-L1 antibody, which blocks PD1/PD-L1 interaction, did not change PD-L1 protein level in both cytosolic/membrane fraction or nuclear fraction (Supplementary information, Fig. S1I). This might explain the lack of inhibition of cell proliferation when the cells were treated with PD-L1 blocking antibody (Supplementary information, Fig. S1j, k).

It has previously been reported that PD-L1 is N-glycosylated and this product has a much higher molecular weight at $50-70 \mathrm{kD}$ detected by Western blot. ${ }^{34}$ PNGase F (peptide-N-glycosidase F) treatment can release the $\mathrm{N}$-glycans attached to asparagine residues on PD-L1 and shift the band down to a lower molecular weight, $\sim 35 \mathrm{kD}$. When we treated both nuclear and cytosolic/ membrane fractions with PNGase F, we found that PD-L1 protein in both fractions shifted down to $\sim 35 \mathrm{kD}$ (Fig. 2e), suggesting that nuclear PD-L1 is a full length protein and is more highly glycosylated compared to the cytosolic/membrane form. Glycosylation of substrates in the Golgi is reported to assist nuclear localization of proteins. ${ }^{35}$ Therefore, these results suggest that PDL1 might be directly transported into the nucleus after being synthesized and glycosylated in the ER and Golgi apparatus.

Interestingly, we noticed a significant increase in the number of multinucleated cells after PD-L1 knockdown (Fig. 2f, g), but not after PD1 depletion (Supplementary information, Fig. S2a). Double knockdown of both PD1 and PD-L1 in MDA-MB-231 cells did not further increase the proportion of multinucleated cells (Supplementary information, Fig. S2b). Analysis of the chromosome structure by chromosome spreads assay showed that the kinetochore region of sister chromatids in control cells were easily detectable. However, in the PD-L1 knockdown cells, approximately $50 \%$ of sister chromatid arms were partially separated and total loss of cohesion between the two sister chromatids was observed in a fraction of cells (Fig. $2 \mathrm{~h}$, i). To analyze sister chromatid cohesion, FISH assay was performed and the distance between the paired myb gene was measured as described previously. ${ }^{26}$ Compared to control cells, the average inter-chromatid distance in PD-L1 depleted cells was much wider, 
a

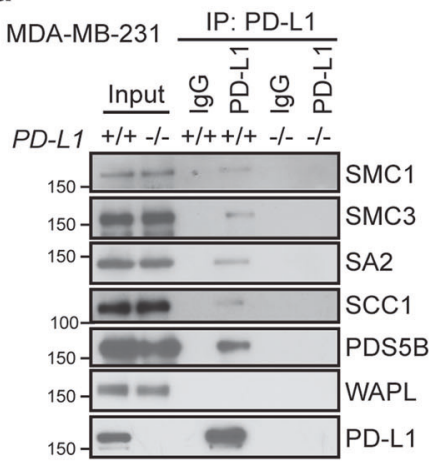

b

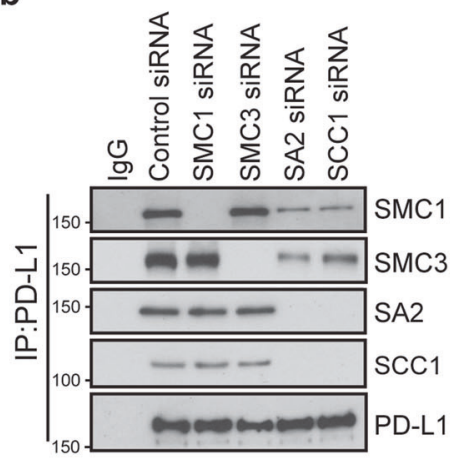

C

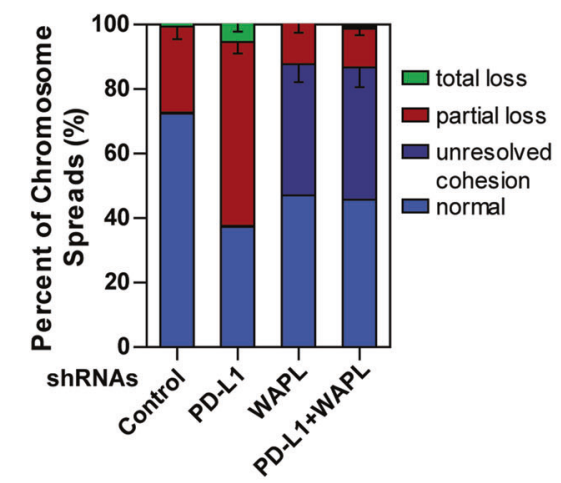

In vitro assay

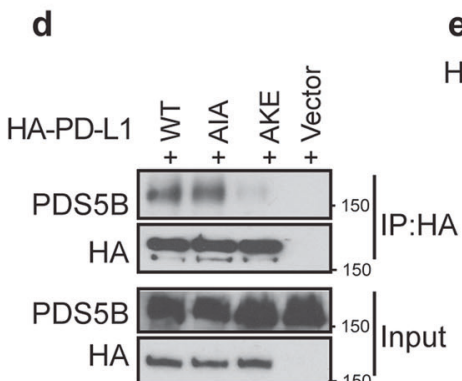

f

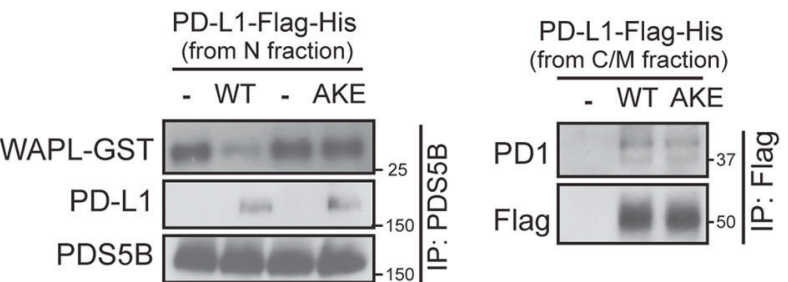

h
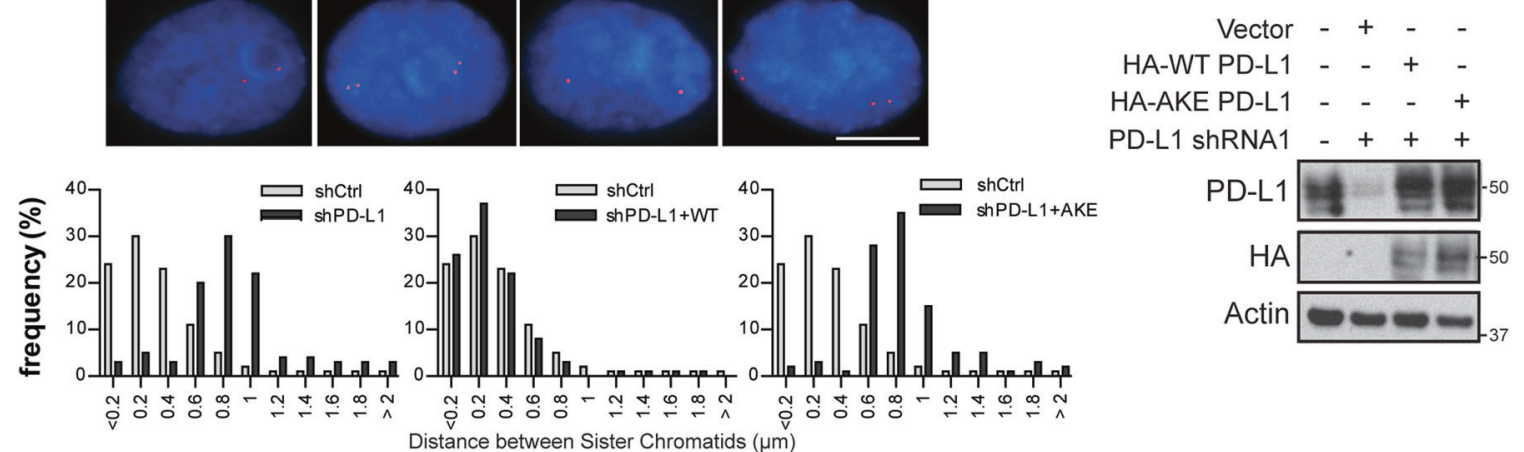

i
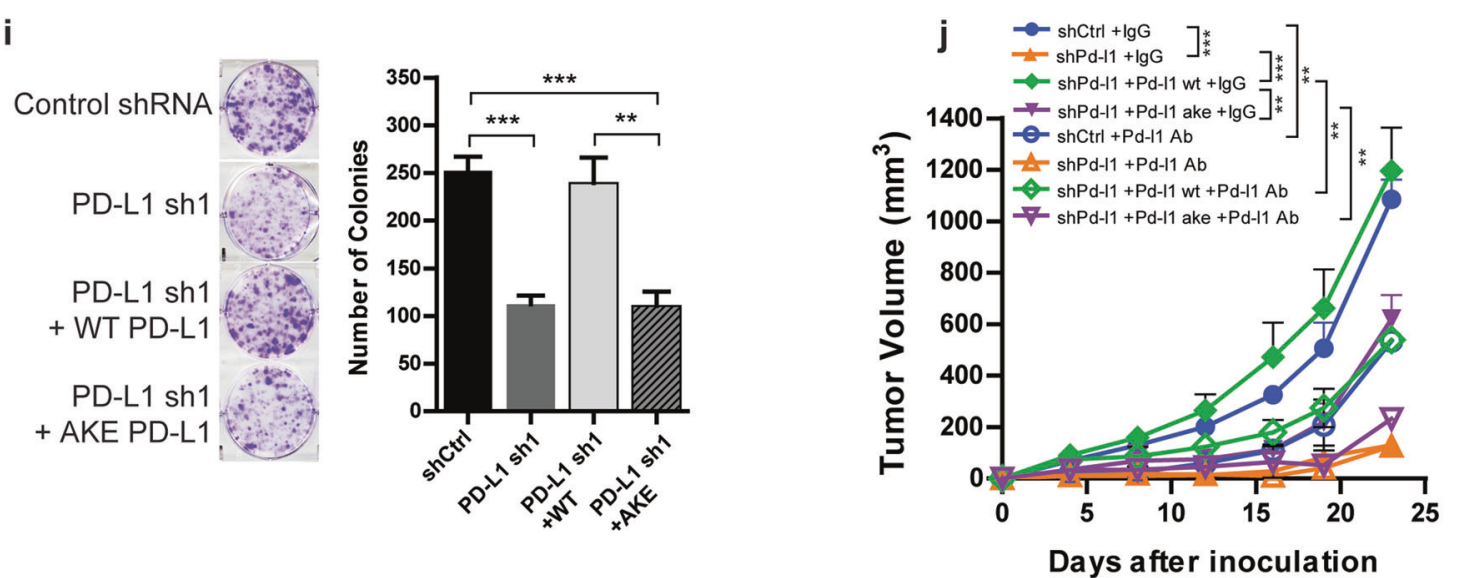

a phenotype similar to that observed after depletion of SCC1, one of the components of the cohesin complex (Fig. 2j, k). Consistent results were detected in three additional $P D-L 1$ knockout cells from different tumor types (Supplementary information, Fig. S3a-c). Collectively, these results suggest that PD-L1 may be involved in regulation of the cohesin complex in cancer cells.

PD-L1 interacts with cohesin complex

The cohesin complex contains four core subunits, SMC1, SMC3, SA2 and SCC1 (also known as RAD21) and forms a ring-shaped structure that traps DNA inside. ${ }^{36}$ The cohesin complex secures correct sister-chromatid segregation during mitosis and meiosis and maintains genome integrity. ${ }^{37-39}$ WAPL drives cohesin release 
Fig. 3 Nuclear PD-L1 interacts with cohesion complex. a, b PD-L1 interacts with cohesin complex. a Doxycycline-inducible PD-L1 widetype (WT) $(+/+)$ and knockout (KO) (-/-) MDA-MB-231 cells were harvested and fractionated. The nuclear fraction was incubated with monoclonal anti-PD-L1 antibody or corresponding IgG. The immunoprecipitates were blotted with indicated antibodies. b Cells were blocked with thymidine and transfected with indicated siRNAs. After releasing from the second round of thymidine block, cells were released into $S$ phase in the presence of MG132 for $3 \mathrm{~h}$. Cells were harvested and nuclear fractions were processed for immunoprecipitation. c PD-L1 is involved in the regulation of cohesion in the presence of WAPL. MDA-MB-231 cells expressing control shRNA, PD-L1 shRNA, WAPL shRNA, or a combination of PD-L1 and WAPL shRNAs were fixed and dropped on coverslips. Percentages of cells with different sister chromatid cohesion status were quantified. Data are presented as means \pm SEM, and were independently replicated three times. $\mathbf{d}-\mathbf{f}$ PD-L1 YSR-like motif (YKR) is essential for PD-L1 binding with PDS5B in vivo, and competes with WAPL for binding to PDS5B in vitro. PD-L1 wild-type (WT), PD-L1 AIA mutant (mutating both FGF-like motifs to AIA), and PD-L1 AKE mutant (mutating YKR to AKE) plasmids were transfected into cells and nuclear fractions were isolated and incubated with (d) HA-conjugated sepharose beads of (e) protein A beads conjugated with anti-PDS5B antibody. The immunoprecipitates were blotted with indicated antibodies. $\mathbf{f}$ Left panel: in vitro competition of binding affinity of PDS5B (purified from insect cells) and purified WAPL (1-30)-GST and PD-L1 (purified from nuclear fraction of MDA-MB-231 cells). Right panel: in vitro interactions between PD1 and wildtype (WT) or AKE mutant PD-L1 purified from cytoplasmic/membrane fraction (C/M) of MDA-MB-231 cells. g-j PD-L1 YSR-like motif is essential for sister chromatid cohesion and cell proliferation. myb gene distance between paired FISH signals ( $\mathbf{g}$ ), and colony formation (i) were measured in MDA-MB-231 cells infected with control shRNA, PD-L1 shRNA, PD-L1 shRNA plus WT shRNA resistant PD-L1 overexpression construct, or PD-L1 shRNA plus shRNA resistant AKE mutant PD-L1 overexpression construct. Scale bar: $10 \mu$ m. Data are presented as means \pm SEM, and were independently replicated three times with similar results. Statistical significance was calculated using Student's $t$-test. ${ }^{* *} P<0.01,{ }^{* * *} P<0.001$. h Protein levels in cells used for assays in $(\mathbf{g}$ and $\mathbf{i})$ were detected by Western blot with indicated antibodies. j B16F10 cells expressing control shRNA (shCtrl), mouse Pd-I1 shRNA (shPd-I1), Pd-I1 shRNA plus shRNA resistant mouse wildtype Pd-I1 (shPd-I1 + Pd-I1 wt), or mouse Pd-I1 shRNA plus shRNA resistant ake mutant Pd-I1 (shPd-I1 + Pd-I1 ake) were injected into C57BL/6 mice. Mice were treated with $5 \mathrm{mg} / \mathrm{kg}$ mouse Pd-l1 blocking antibody or corresponding IgG. The tumor growth was monitored by measuring with a caliper every 3-4 days. Data are presented as means \pm SEM $(\mathrm{n}=5)$. Two-way ANOVA was used for the comparisons. ${ }^{* *} P<0.01,{ }^{* * *} P<0.001$.

from chromatin by opening a distinct DNA exit gate at the interface connecting the SMC3 and SCC1 subunits. ${ }^{40,41}$ On the other hand, Sororin antagonizes WAPL and maintains sister chromatid cohesion. ${ }^{26}$ Based on the above observations, we were interested in testing whether PD-L1 might be directly connected to the cohesin complex. Specifically, we carried out immunoprecipitation assays and examined interactions between nuclear PDL1 and the cohesin complex in the nuclear fraction. We detected all four subunits of the cohesin complex, SMC1, SMC3, SA2, and SCC1, in the PD-L1 immunoprecipitates, but not in IgG or PD-L1 knockout immunoprecipitates (Fig. 3a). Similar interactions were also detected in additional cancer cell lines expressing high levels of PD-L1 (Supplementary information, Fig. S4a). To determine which subunit of the cohesin complex directly interacts with nuclear PD-L1, we depleted each individual subunit by using siRNA and pulled down nuclear PD-L1 to detect interacting proteins. When we knocked down SMC1 or SMC3 in cells with specific siRNAs, the interaction of PD-L1 with the other subunits did not change. However, when we depleted SA2 or SCC1, PD-L1 interaction with the cohesin complex was disrupted (Fig. 3b and Supplementary information, Fig. S4b), indicating that PD-L1 interacts with the cohesin complex through the SA2 and SCC1 subunits. Importantly, knocking down individual components of the cohesin complex did not affect the expression of the remainder of the complex (Supplementary information, Fig. S4b)

WAPL is the protein responsible for opening the hinge of the cohesin complex and maintaining the dynamic binding state. Overexpression of WAPL causes cohesion defects similar to what we observed in PD-L1 depleted cells. ${ }^{25,42}$ To determine the relationship between WAPL and PD-L1, we knocked down WAPL and PD-L1 both separately and simultaneously, and examined the chromosome cohesion. As expected, knockdown of WAPL led to tightly connected sister chromatids. Depletion of WAPL and PD-L1 simultaneously caused a phenotype similar to WAPL depletion alone (Fig. 3C), suggesting that PD-L1 regulates cohesion in the presence of WAPL. However, we did not detect an interaction between PD-L1 and WAPL. Surprisingly, PDS5B, a competitive binding partner for both WAPL and Sororin, was detected in the PD-L1 immunoprecipitates (Fig. 3a). Based on these observations, we hypothesized that PD-L1 might compete with WAPL for its binding to PDS5B. Two PDS5B binding motifs, the FGF motif and the YSR motif, have been previously identified. ${ }^{24,26,43}$ We examined the protein sequence of PD-L1 and found two FGFlike motifs and one YSR-like motif (Supplementary information, Fig. S4c, d). Unlike the FGF-like motif, the YSR-like motif was conserved across species. While mutation of the FGF-like motif did not affect the binding between PD-L1 and PDS5B, mutating the highly conserved YKR residues within the YSR-like motif to AKE (amino acids YKR mutated into AKE) greatly decreased the binding of PD-L1 to PDS5B (Fig. 3d). We also observed that wild-type PD-L1 expression decreased the interaction between PDS5B and WAPL, while the PD-L1 AKE mutant did not (Fig. 3e). This suggests a competition between PD-L1 and WAPL in binding to PDS5B. In vitro competition assays showed that purified wildtype PD-L1, but not the AKE mutant from nuclear fraction, was able to compete with WAPL for its binding to PDS5B (Fig. 3f). Meanwhile, both the wild-type PD-L1 and purified AKE mutant from the cytosolic/ membrane fraction interacted with PD1 (Fig. 3f, right panel), further supporting our finding that the nuclear function of PD-L1 is independent of its function as the ligand for PD1 (Fig. 1e-I). To further evaluate this competition functionally, we reintroduced WT PD-L1 or the AKE mutant into PD-L1 knockdown cells, and found that expression of WT PD-L1, but not the mutant PD-L1, restored sister chromatids cohesion, cell proliferation, and colony formation (Fig. $3 \mathrm{~g}-\mathrm{i}$ ). Similar data were obtained using the PD-L1 knockout MDA-MB-231 cells (Supplementary information, Fig. S4e), suggesting that PD-L1 competes with WAPL binding to PDS5B and enhances sister chromatid cohesion. To further test whether the AKE mutation affects tumor growth and the immune suppression function of PD-L1, we injected mouse B16F10 cells expressing control shRNA, mouse Pd-I1 shRNA, mouse Pd-I1 shRNA plus shRNA resistant wildtype $\mathrm{Pd}-\mathrm{I1}$, or mouse $\mathrm{Pd}$-I1 shRNA plus shRNA resistant ake $\mathrm{Pd}-11$ into immunocompetent $\mathrm{C} 57 \mathrm{BL} / 6$ mice. These mice were treated with mouse Pd-I1 blocking antibody or control IgG antibody. Consistent with the results obtained in MDA-MB-231 xenografts (Fig. 1d), loss of Pd-I1 suppressed tumor growth (Fig. 3j). Restoration of wildtype Pd-11 rescued tumor growth. Consistent with the colony formation results obtained in human cancer cells, reintroduction of the ake mutant impaired tumor growth when compared to the wildtype Pd-11 expressing xenografts (Fig. 3j). Furthermore, mouse Pd-I1 blocking antibody suppressed the growth of tumor expressing control shRNA, but had no effect on Pd-11 depleted tumors (Fig. 3j). Consistently, this blocking antibody inhibited tumor growth in $\mathrm{Pd}-11$ depleted xenografts restored with the wildtype Pd-I1 or the ake mutant, regardless of the fact that xenograft tumors expressing wildtype Pd-11 grew much faster than tumors expressing the ake mutant (Fig. 3j and Supplementary information, Fig. S5a, b). These results suggest that the PD-L1 AKE mutant impairs PD-L1 function in the nucleus while still maintaining its immunosuppressive effect. 


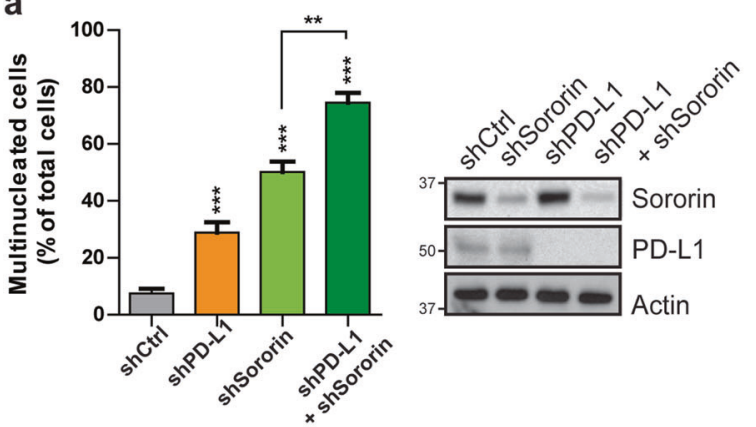

C

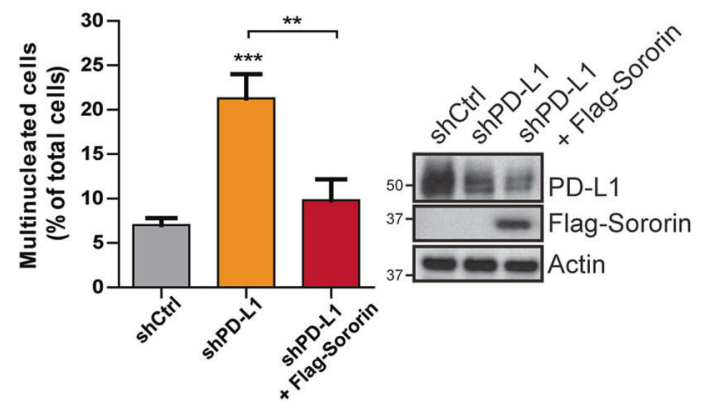

e

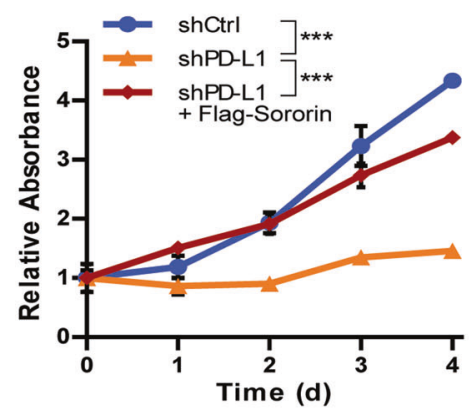

g

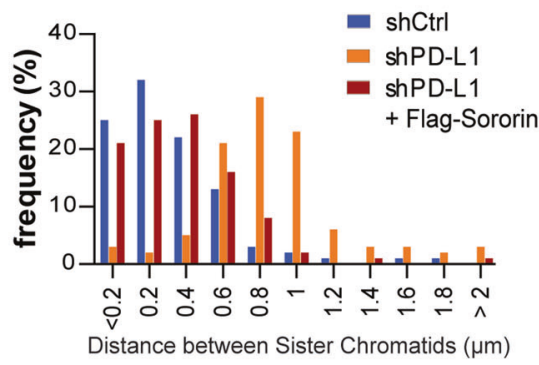

b

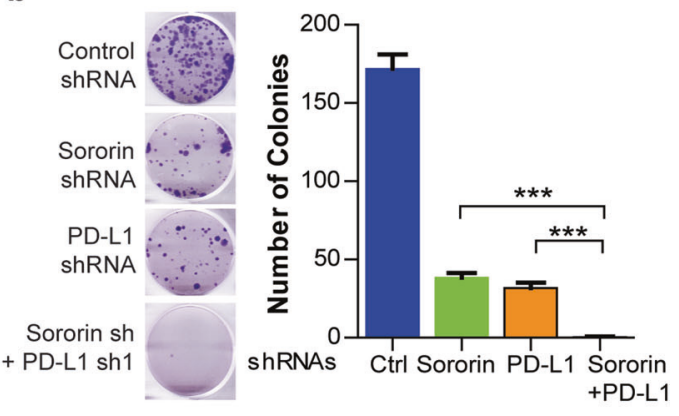

d
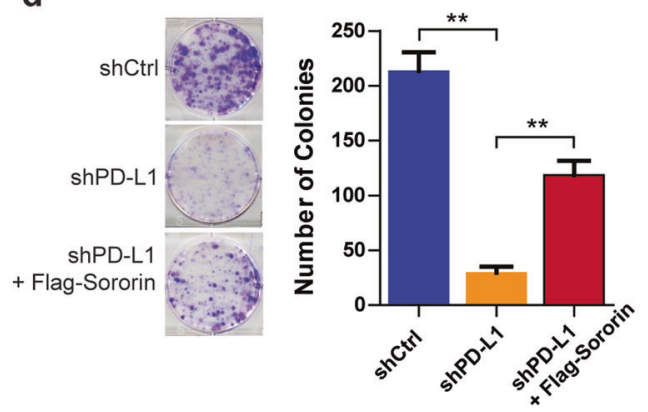

f

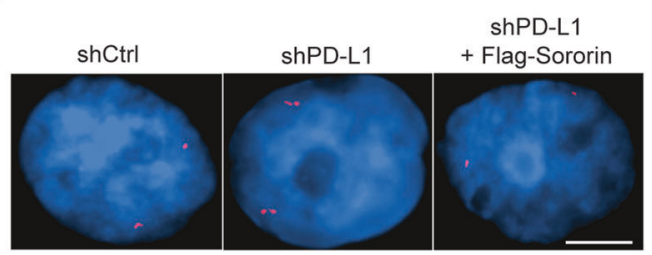

h

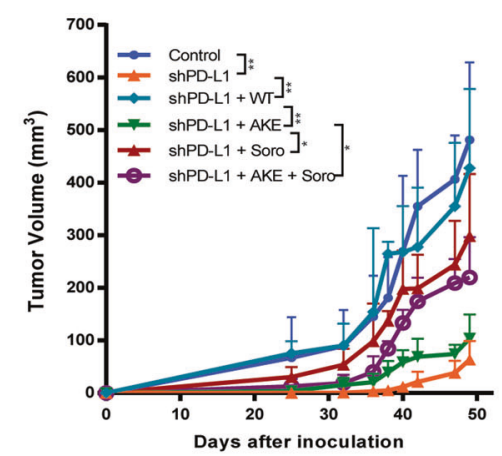

Fig. 4 PD-L1 compensates for the loss of Sororin and regulates sister chromatid cohesion. a, b Effect of double knockdown of PD-L1 and Sororin on various cellular phenotypes in MDA-MB-231 cells. Quantification of multinucleated cells (a) and colony formation (b) in cells expressing control shRNA, PD-L1 shRNA, Sororin shRNA, or combined PD-L1 shRNA and Sororin shRNA, respectively. c-h Overexpression of Sororin reversed various cellular phenotypes caused by depletion of PD-L1 in MDA-MB-231 cells. Quantification of multinucleated cells (c), colony formation (d), cell proliferation (e), representative images of myb gene distance between paired FISH signals (f), and quantification of myb gene distance between paired FISH signals (g), tumor growth in NSG mice xenografted with cells expressing control shRNA, PD-L1 shRNA, PD-L1 shRNA plus WT or AKE PD-L1, PD-L1 shRNA plus Sororin, or PD-L1 shRNA + Sororin + AKE PD-L1 expressing plasmid (h). For cell experiments, data are presented as means \pm SEM, and were independently replicated three times with similar results. Statistical significance was calculated using 2-way ANOVA in e. Statistical significance was calculated using Student's t-test in a-d. For experiments related to the xenograft mice models, tumor growth data are presented as means $\pm S D(n=5)$ and 2-way ANOVA was used for the comparisons in $\mathbf{h}$. ${ }^{*} P<$ $0.05,{ }^{* *} P<0.01,{ }^{* * *} P<0.001$.

PD-L1 compensates for the loss of Sororin and regulates sister chromatid cohesion

The function of PD-L1 is reminiscent of that of Sororin-to compete with WAPL for binding to PDS5B and to stabilize cohesion on DNA. ${ }^{28}$ To further determine the biological implication of this observation, we first examined the expression of Sororin in breast cancer cell lines of different subtypes. Surprisingly, Sororin was more highly expressed in Luminal A, Luminal B, and HER2 + cells and less well expressed in TNBC cells, a pattern opposite to that of PD-L1 (Supplementary information, 
a

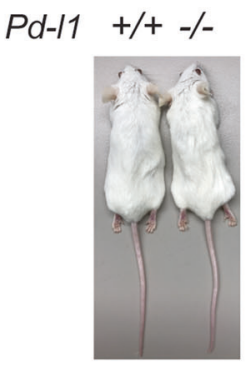

C

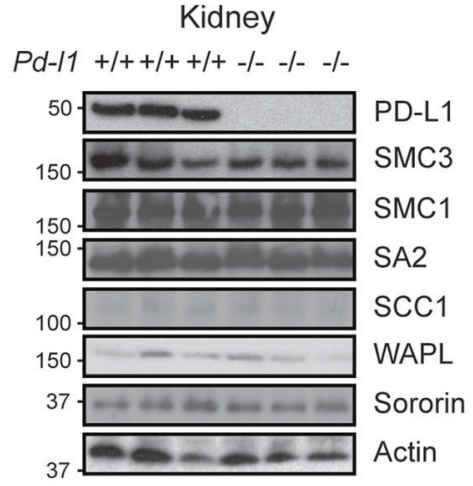

b

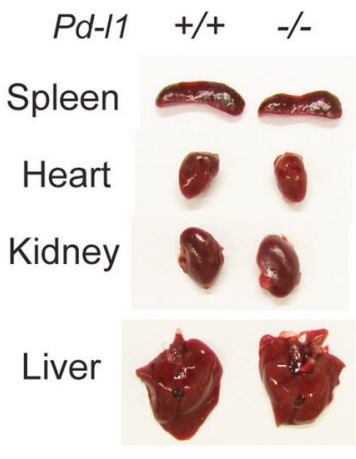

Liver

Pd-l1 +/++/+ +/+ -/- -/- -/-

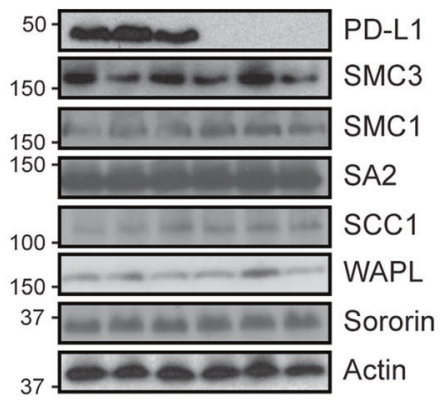

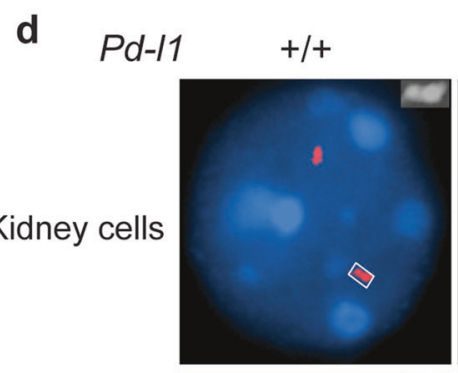

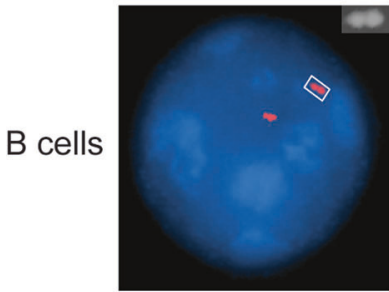

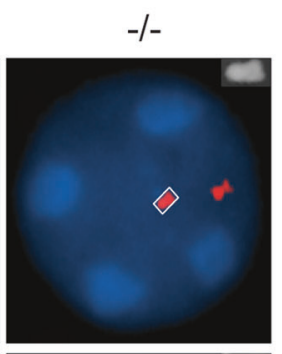

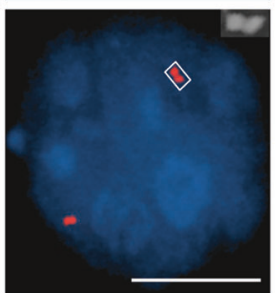

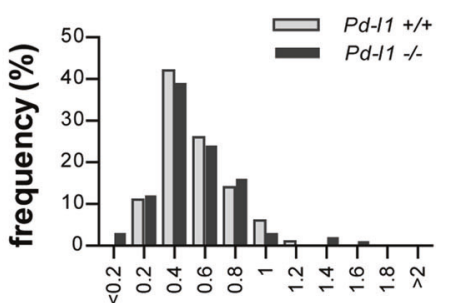

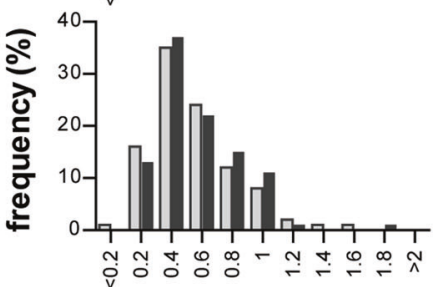

Distance between Sister Chromatids ( $\mu \mathrm{m})$

Fig. 5 Pd-I1 knockout mice display normal phenotypes including sister chromatid cohesion. a Image of $P d-/ 1 \mathrm{WT}(+/+)$ and KO (-/-) mice. b Comparison of organ sizes between the $P d-I 1$ WT $(+/+)$ and $\mathrm{KO}(-/-)$ mice. c Protein expression of cohesin subunits in kidney or liver tissues from the $P d-I 1$ WT $(+/+)$ and KO $(-/-)$ mice. d Representative images of FISH assays to determine sister chromatid cohesion in primary kidney cells and B cells isolated from the $P d-11 \mathrm{WT}(+/+)$ and $\mathrm{KO}(-/-)$ mice. Scale bar: $10 \mu \mathrm{m}$.

Fig. S1c). We also determined PD-L1 and Sororin expression in human breast cancer tissues by tissue microarray (TMA), and found that tumor PD-L1 expression was negatively correlated with Sororin expression in TNBCs (Supplementary information, Fig. S6a-c). Based on these observations, we hypothesized that PD-L1 may regulate chromatid cohesion and compensate for the defect caused by the loss of Sororin.

To test this hypothesis, we knocked down PD-L1 and Sororin separately or together and examined the number of multinucleated cells. We found that depletion of Sororin or PD-L1 individually greatly induced the formation of multinucleated cells. Depletion of both proteins simultaneously resulted in even more multinucleated cells when compared to individual knockdowns (Fig. 4a). We also performed colony formation assay, and found that knockdown of both PD-L1 and Sororin showed a greater suppression of colony formation when compared with single gene knockdown (Fig. 4b). Taken together, these results suggest that PD-L1 and Sororin may function independently.

To further confirm the independent roles of PD-L1 and Sororin, we overexpressed Sororin in PD-L1 knockdown MDA-MB-231 cells, and found that introducing Sororin decreases the number of multinucleated cells caused by the loss of PD-L1 (Fig. 4c). We further observed restoration of cell proliferation, colony formation, as well as sister chromatid cohesion upon overexpression of 
Sororin in PD-L1 depleted MDA-MB-231 cells (Fig. 4d-g). We also injected NSG mice with control cells, PD-L1 knockdown cells, and PD-L1 knockdown cells ectopically expressing Sororin. Consistently, loss of PD-L1 significantly impaired tumor growth, but overexpression of Sororin alleviated this impairment (Fig. 4h), suggesting that PD-L1 compensates for the function of Sororin in TNBC cells, which have low Sororin expression. Finally, we also ectopically expressed PD-L1 in Sororin depleted BT474 cells. As expected, we found that the loss of Sororin greatly suppressed colony formation, while the introduction of PD-L1 rescued this growth defect (Supplementary information, Fig. S7).

Normal sister chromatid cohesion in $P d-11$ knockout mice To better understand nuclear PD-L1 function in normal cell homeostasis, we utilized $P d-11$ knockout mice. ${ }^{44}$ We did not observe a significant growth defect in $P d-11$ knockout mice (Fig. 5a, b). We also did not find any changes in protein levels of cohesin complex subunits in $P d-11$ knockout mice when compared to wildtype mice (Fig. 5c). Moreover, FISH assay using primary mouse cells did not reveal significant changes in the distance between sister chromatids (Fig. $5 \mathrm{~d}$ ). These results suggest that PD-L1 may not be involved in cohesion regulation in normal cells. This could be due to the fact that Sororin is maintained at normal protein levels under normal conditions.

\section{DISCUSSION}

Previous studies have reported that tumor PD-L1 interacts with its receptor PD1, suppresses the development of the $T$ cell response and evades anti-tumor immunity. ${ }^{6,7}$ Moreover, increasing evidences demonstrate that both PD-L1 and PD1 also play intrinsic roles in cancer cell signaling. Intrinsic PD1 in melanoma cells regulates mTOR signaling and promotes tumor growth. ${ }^{32}$ Intrinsic PD-L1 is also implicated to be involved in different biological processes, such as autophagy and glucose metabolism. ${ }^{14-16}$ Here we found that PD-L1 is overexpressed in multiple different cancers including triple negative breast cancer, which portends a poor prognosis (Supplementary information, Fig. S1c). In addition, we also detected nuclear localization of PD-L1 protein (Fig. 2b, c). In spite of extensive studies on its function as a cell membrane protein, little is known about PD-L1 as a nuclear protein. The membrane protein detected in the nucleus is not an exception for PD-L1. There is a substantial body of evidence documenting other membrane proteins e.g. epidermal growth factor receptor (EGFR) family members to be localized in the nucleus. ${ }^{45,46}$ The translocation of EGFR family members involves the recognition of the nuclear localization signal (NLS) by the nuclear transporter importin proteins, thus directing the proteins transport through the nuclear pore complex to the nucleus. ${ }^{47-49}$ However, unlike EGFR, PD-L1 does not contain a nuclear localization sequence. Post-translation modifications in the ER and trans-Golgi network, including glycosylation, have been implicated in trafficking of various proteins to the plasma membrane or cell nucleus. ${ }^{50,51}$ Previous studies and the current study have shown that PD-L1 is exclusively $\mathrm{N}$-glycosylated (Fig. 2e). ${ }^{34}$ Compared to the plasma membrane PD-L1 ( $50 \mathrm{kD}$ shown on immunoblot), the nuclear PDL1 (>150 kD on immunoblot) may involve more complex glycan forms (Fig. 2c-e), suggesting that nuclear PD-L1 is less likely directly internalized from plasma membrane. It is possible that PDL1 is heavily glycosylated after translation in the ER and Golgi apparatus, and that this heavily glycosylated form of PD-L1 is recognized by glycosylation binding proteins and transported into the nucleus. The exact mechanisms involved in the process of nuclear PD-L1 localization requires further investigation.

Here we report that nuclear PD-L1 interacts with cohesin complex and regulates sister chromatid cohesion (Figs. $2 \mathrm{~h}-\mathrm{i}$ and $3 a-g)$. Mechanistically, nuclear PD-L1 competes with WAPL for its binding to PDS5B through its YSR like motif and stabilizes the cohesin ring (Fig. 3d-f). This process is reminiscent of Sororin which also contains a YSR motif and competes with WAPL for binding to PDS5B. ${ }^{43}$ Another protein, Haispin also contains a similar motif and protects centromeric cohesion by antagonizing WAPL. ${ }^{52}$ In our study, we have identified that nuclear PD-L1 functions similarly to Sororin to protect sister chromatid cohesion and prevent cell death from lack of Sororin. Loss of PD-L1 greatly impairs sister chromatid cohesion (Figs. $2 \mathrm{~h}-\mathrm{j}$ and $3 \mathrm{a}$ ) and suppresses tumor growth (Figs. 1a-d and 3j). These phenotypes are not limited to triple negative breast cancer and are also detected in other cancer cells from different tissue origins with high PD-L1 expression (Fig. 2d; Supplementary information, Figs. S1h, S3, and S4a). Data from Pd-11 knockout mouse model show that PD-L1 is not essential for the maintenance of normal tissue homeostasis (Fig. 4a, b). Disruption of PD-L1 does not affect chromatid cohesion function due to normal Sororin protein level (Supplementary information, Fig. S4c, d).

YSR like motif is localized in the PD-L1 extracellular domain. This domain is essential for the interaction between PD-L1 and its receptor, PD1 and immune checkpoint signaling. ${ }^{53}$ However, we found that mutation of YSR like motif did not affect membrane PD-L1 interaction with PD1 (Fig. 3f), and the mutant PD-L1 still maintained PD-L1 immunosuppression effect in vivo (Fig. 3j; Supplementary information, Fig. S5a, b).

Based on these findings, we propose a working model that in cancer cells with normal Sororin expression, Sororin antagonizes WAPL and controls proper sister chromatid cohesion (Fig. 6). In contrast, in TNBC or other cancers with low Sororin expression, $\mathrm{PD}-\mathrm{L} 1$ overexpression is one of the mechanisms that compensates for the loss of Sororin. PD-L1 antagonizes WAPL binding to PDS5B in the cohesin complex, leading to proper chromosome segregation and cell proliferation. This observation can be extended to other cancers such as lung, colon and prostate cancers, in which the PD-L1-cohesion axis also exists. This might be one of the adaptive mechanisms for Sororin-low cancers to maintain cell proliferation, and at the same time, evade immune surveillance.

Taken together, we have reported an important and novel function of nuclear PD-L1 in the regulation of chromatid cohesion. Nuclear PD-L1 is important for establishment of the cohesin complex, controlling proper chromosome segregation and maintaining genomic integrity. Targeting nuclear PD-L1, which does

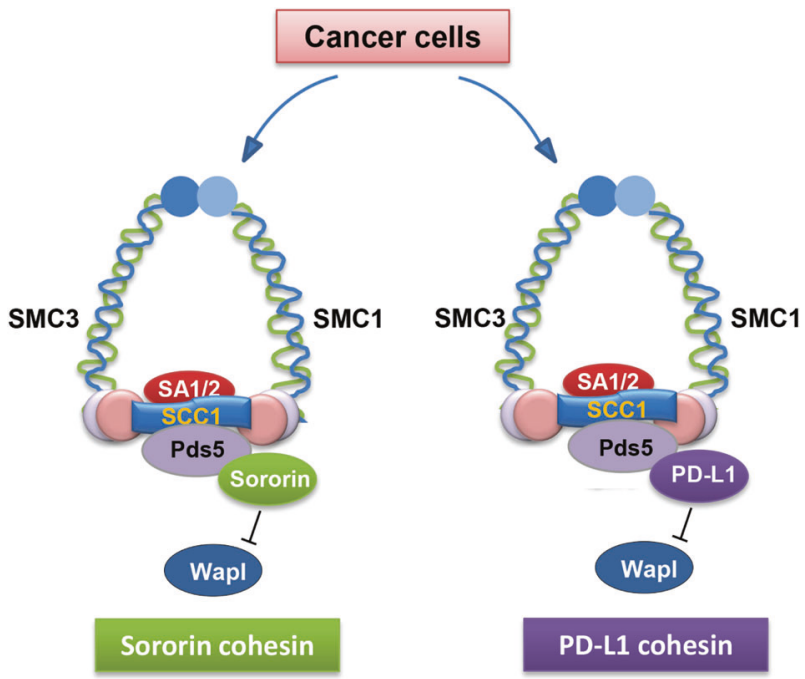

Fig. 6 Working model. Sororin suppresses WAPL and maintains sister chromatid cohesion in Sororin cohesin cells. In contrast, in PDL1 overexpressed cells, PD-L1 compensates for the loss of Sororin and competes with WAPL binding to PDS5B, thereby regulating sister chromatid cohesion. 
not affect normal cells, may clinically benefit cancer patients with high tumor PD-L1 levels.

\section{METHODS}

Cell culture, synchronization and transfection

The cell lines MDA-MB-231, Hs 578T, MCF7, ZR75-1, BT474, BT-549, RKO, HCC837, B16F10 and DU145 were cultured in DMEM or RPMI1640 containing 10\% FBS. Breast cancer cells expressing shRNAs were generated by infecting the cells with individual shRNA virus followed by selecting resistant clones in growth medium supplemented with puromycin. Rescue experiments were conducted by transducing both shRNA lentivirus and shRNA resistant CDS expressing lentivirus into cells and selecting with puromycin for one day. For synchronization, MD-MB-231 cells were cultured in the presence of $2 \mathrm{mM}$ thymidine (Sigma) for $24 \mathrm{~h}$, washed twice with pre-warmed PBS and released in a fresh medium for $8 \mathrm{~h}$. Thymidine was added again to a final concentration of $2 \mathrm{mM}$ to block cells at G1/S. After another $16 \mathrm{~h}$ incubation, cells were released. For Scc1 and Sororin knockdown, $30 \mathrm{nM}$ (single RNAi experiments) or $60 \mathrm{nM}$ (double RNAi experiments) siRNAs were pre-mixed with Lipofectamine RNAiMax (Life Technologies) according to the manufacturer's instructions and were added either before the first thymidine arrest (Scc1) or during the first release (Sororin), as described previously. ${ }^{26}$

Plasmids and reagents

PD-L1 sgRNA plasmids and inducible Cas9 expression plasmids were purchased from Dharmacon. Mouse Wildtype and AKE mutant PD-L1 coding sequences were inserted into the mouse pGIPZ-PD-L1 shRNA plasmid to replace the GFP coding sequencing. Flag-PD-L1 plasmids were purchased from Origene. Point mutations in PD-L1 were generated using the Quick-change sitedirected mutagenesis kit (Stratagene) and sequences were verified by Sanger sequencing. Expression plasmids for various proteins were cloned into a pCMV plasmid (Clontech Laboratories, Inc.) with a hemagglutinin (HA) tag. Sororin plasmid was purchased from Origene.

Anti-SMC1 antibody (4802S), anti-SMC3 antibody (5696S), antiSCC1 antibody (4321S), anti-STAG2 antibody (5882S), anti-WAPL antibody (77428S) and anti-PD-L1 antibody (13684S, for Western blot) were purchased from Cell Signaling. Anti-PD-L1 antibody (29E.2A3) for immunoprecipitation and human cell PD-L1 blocking antibody were purchased from Biolegend. Anti-Sororin antibody (ab192237), anti-SMC1 antibody (ab140493), anti-Na/K ATPase antibody (Ab76020), anti-CENPA antibody (ab13939) and anti-PD1 antibody (ab52587) were purchased from Abcam. Anti-CDH1 antibody (sc-56312), anti-CDC20 antibody (sc-136024), anti-cyclin E antibody (sc-271348), anti-PCNA antibody (sc-56) and anti-cyclin B1 antibody (sc-70898) were purchased from Santa Cruz. Anti- $\beta$ actin antibody was purchased from Sigma. Anti-PDS5B antibody was purchased from Bethyl laboratories; anti-mouse PD-L1 blocking antibody (10F.9G2) and corresponding IgG were purchased from Bioxcell.

Generation of inducible $P D-L 1$ knockout cells

MDA-MB-231 cells were infected with inducible Cas9 plasmid and selected with $5 \mu \mathrm{g} / \mathrm{mL}$ blasticidin for two weeks in Tet-free medium. The cells were then infected with $P D-L 1$ sgRNA lentivirus and selected with both blasticidin and puromycin. Single colonies were picked up and screened for $P D-L 1$ knockout with doxycycline induction.

Animals

All animal work was approved by the Mayo Clinic Institutional Animal Care and Use Committee (IACUC). 6-8 week-old female immune-deficient NSG (NOD.Cg-Prkdcscid $\| 2 \mathrm{rgtm} 1 \mathrm{Wjl} / \mathrm{SzJ}$ ) mice and 6-8 week-old female C57BL/6 mice were ordered from
Jackson Laboratories. $P d-/ 1$ knockout mice were maintained in a $12 \mathrm{~h}$ light/dark cycle, and fed ad libitum normal food.

Xenograft models

MDA-MB-231 cells stably expressing control shRNA, shRNAs targeting PD-L1 or PD1 genes, or cells overexpressing PD-L1 or indicated genes were mixed in PBS 1:1 with matrigel (BD Bioscience). Cells $\left(1 \times 10^{6}\right.$ cells/mouse $)$ were then injected subcutaneously into 6-8 week-old female immune-deficient NSG (NOD.Cg-Prkdcscid II2rgtm1Wjl/SzJ) mice. B16F10 stably expressing control shRNA, mouse Pd-I1 shRNA, mouse Pd-I1 shRNA plus shRNA resistant wildtype $\mathrm{Pd}-\mathrm{I1}$, or mouse $\mathrm{Pd}-\mathrm{I1}$ shRNA plus shRNA resistant ake mutant $\mathrm{Pd}-11$ were injected subcutaneously into C57BL/6 mice and were treated with $5 \mathrm{mg} / \mathrm{kg}$ mouse Pd-I1 blocking antibody twice a week. Tumors were measured with a caliper on the indicated days. Tumor volumes were calculated as described previously. ${ }^{54,55}$ Mice were sacrificed and tumors were dissected when they met the criteria set by IACUC. Data were analyzed using ANOVA or Student's $t$-test.

\section{Clonogenic assay}

For clonogenic assays, breast cancer cells were seeded in triplicate in 6-well plates at 500 or 1000 cells per well. Ten to 15 days later, cells were fixed with $100 \%$ methanol for $5 \mathrm{~min}$, followed by $0.1 \%$ (w/v) Giemsa. Colonies with more than 50 clones were counted on a GelCount (Oxford Optronix).

Fluorescence in situ hybridization (FISH)

Prior to processing, slides were aged on a $90^{\circ} \mathrm{C}$ ThermoBrite ${ }^{\mathrm{TM}}$ for 5 to $10 \mathrm{~min}$. Home-brewed human MYB DNA clones $(521 \mathrm{H} 20$, RP132B1, RP3-388E23 and RP1-71N10) or mouse clones, 4qE2 (RP23182D5, RP23-149J22, RP24-256N4, RP24-231113) probe cocktail were used for FISH to detect sister chromatids' signals in human or mouse cells. Probes labeled with SpectrumOrange dUTP (Abbott Molecular/Vysis Products) were combined into one probe, respectively. The probe was then applied onto individual slides, hybridized, and washed according to the Interphase FISH protocol. Specifically, $5-10 \mu \mathrm{L}$ of the DNA probe working solution was applied to selected hybridization areas on the pre-treated slides, and then coverslipped and denatured using ThermoBrite (Abbott Molecular) at $75^{\circ} \mathrm{C}$ for $5 \mathrm{~min}$, followed by hybridization for $20 \mathrm{~h}$ in a $37^{\circ} \mathrm{C}$ humidified oven.

Post-hybridization wash was performed first with pre-warmed $0.4 \times$ SSC (saline-sodium citrate) for $2 \mathrm{~min}$ at $74^{\circ} \mathrm{C}$, followed by $0.1 \% \mathrm{NP}-40 / 2 \times \mathrm{SSC}$ solution in a dark environment at room temperature for $1 \mathrm{~min}$. The slides were stained with DAPI, 4'-6,diamidino-2-phenylindole (Vector Laboratories, Burlingame, CA) and coverslipped. Visualization and acquisition of images of the FISH signals were performed by Cytovision (Leica Biosystems, Buffalo Grove, Illinois). FISH signals representing distance between sister chromatids were measured in Image J. On average, more than 200 cells were measured and counted for each experiment.

Cell fractionation assay

For chromatin isolation, cells were harvested and lysed with 10 volumes of NETN buffer with low salt $(20 \mathrm{mM}$ Tris-HCl, $\mathrm{pH} 8.0,10$ $\mathrm{mM} \mathrm{NaCl}, 1.5 \mathrm{mM} \mathrm{MgCl}, 1 \mathrm{mM}$ EDTA, 0.5\% Nonidet P-40, $20 \mathrm{mM}$ $\mathrm{NaF}, 1 \mathrm{mM} \mathrm{Na} \mathrm{VO}_{4}, 1 \mu \mathrm{g} / \mathrm{mL}$ aprotinin, and $1 \mu \mathrm{g} / \mathrm{mL}$ pepstatin). The chromatin-enriched pellet was washed with PBS three times and resuspended in $0.2 \mathrm{M} \mathrm{HCl}$ for $30 \mathrm{~min}$ on ice. The soluble extract was neutralized with $1 \mathrm{M} \mathrm{NaOH}$ for further analysis. Cytoplasmic/ Membrane and nuclear fractions were separated using the Paris Kit (Ambion). Membrane, cytoplasmic and nuclear fractions were isolated by Plasma Membrane Protein Extraction Kit (Abcam).

\section{Chromosome spreading}

Cells were cultured with colcemid solution overnight $(1 \mu \mathrm{g} / \mathrm{mL})$. Cells were harvested by mitotic shake off and mixed with 
hypotonic solution $(0.8 \%$ sodium citrate hypotonic: $0.075 \mathrm{M}$ potassium chloride hypotonic, $\mathrm{pH} 6.0$ ) at room temperature for 20-25 min. The cell mixtures were then washed three times with freshly made Carnoy's solution (methanol to glacial acetic acid 3:1). Cells in Carnoy's solution were then dropped onto glass slides and dried at $37^{\circ} \mathrm{C}$. Slides were then stained with $\mathrm{pH} 6.8,5 \%$ Giemsa (Merck) for 10 min and washed carefully with tap water.

\section{RNA interference}

PD-L1 shRNA sh1 (target sequence GACCTATATGTGGTAGAGTAT) and sh2 (target sequence CGAATTACTGTGAAAGTCAAT), and PD1 shRNA sh1 (target sequence GCCTAGAGAAGTTTCAGGGAA) and sh2 (target sequence (CGTGCTAAACTGGTACCGCAT), were purchased from Sigma. Lentivirus was produced according to manufacturer's protocol. siRNAs targeting SMC1, SMC3, SA2 and SCC1 were purchased from Dharmacon.

Western and Immunoprecipitation (IP) assay

Cells were lysed with $0.5 \%$ NP40, $150 \mathrm{mM} \mathrm{NaCl}, 50 \mathrm{mM}$ Tris, and $1 \mathrm{mM}$ EDTA (NETN) buffer containing $20 \mathrm{mM}$ Tris- $\mathrm{HCl}(\mathrm{pH} 8.0)$, $100 \mathrm{mM} \mathrm{NaCl}, 1 \mathrm{mM}$ EDTA, $0.5 \%$ Nonidet P-40 with $50 \mathrm{mM}$ bglycerophosphate, $10 \mathrm{mM} \mathrm{NaF}$, and $1 \mathrm{mg} / \mathrm{mL}$ each of pepstatin $A$ and aprotinin and sonicated. After centrifugation, the supernatant was removed and incubated with indicated antibodies and protein A or protein $G$ Sepharose beads (Amersham Biosciences) for $2 \mathrm{~h}$ or overnight at $4{ }^{\circ} \mathrm{C} .{ }^{56}$ The samples were then analyzed by SDS-PAGE following three rinses with NETN buffer. Western blots were performed following standard procedures.

\section{Immunofluorescent staining}

Cells were cultured on coverslips, and washed twice with prewarmed PBS. For regular PD-L1 staining, cells were fixed with $3 \%$ paraformaldehyde for $10 \mathrm{~min}$ followed by $0.5 \%$ triton (PFA+ Triton X-100) for $5 \mathrm{~min}$. To better detect the nuclear PD-L1, cells were first permeabilized with $0.5 \%$ Triton $X-100$ for 5 min at room temperature to remove major amount of PD-L1 protein in the cytoplasm and cell membrane, and then fixed with $3 \%$ paraformaldehyde for $10 \mathrm{~min}$. Cells were blocked with $5 \%$ goat serum and then incubated with PD-L1 antibody for $1 \mathrm{~h}$ at room temperature. After washing with PBS three times, Alexa-568conjugated anti-rabbit secondary antibody or Alexa-488conjugated anti-mouse secondary antibody (Jackson ImmunoResearch) was added. This was incubated for $30 \mathrm{~min}$ at room temperature. The cells were then counterstained with DAPI to visualize nuclear DNA. Coverslips were mounted onto glass slides with mounting solution and visualized by fluorescence microscope. The nuclear PD-L1 intensity was quantified using ImageJ software.

\section{Cell proliferation assay}

Cells expressing different shRNAs were seeded in 6-well cell culture plates in triplicate at a density of 5000 cells per well in $2 \mathrm{~mL}$ medium supplemented with $10 \%$ FBS. The medium was changed every day. The cell number at the indicated time points was determined by counting using a hemocytometer.

\section{Statistical analysis}

Data in bar or line graphs are presented as means \pm SD or means \pm SEM of at least three independent experiments. Comparisons were carried out with ANOVA or unpaired Student's $t$-test $\left({ }^{*} P<0.05,{ }^{* *} P<0.01,{ }^{* * *} P<0.001\right)$.

\section{ACKNOWLEDGEMENTS}

We thank the Mayo Cytogenetics Core which is directed by Patricia T. Greipp for performing the FISH assays and for the analysis of those assays. We also thank Drs H. $\mathrm{Yu}$ and M.C. Hung for kindly providing the plasmids. This work was supported by the Mayo Clinic Center for Individualized Medicine, the Mayo Clinic Center for Biomedical
Discovery, the Mayo Clinic Breast SPORE (grant number P50CA 116201-9), and by the National Institutes of Health (grants number CA 196648 to L.W., CA203561 to Z.L.).

\section{AUTHOR CONTRIBUTIONS}

J.Y., B.Q., R.W., Z.L. and L.W. designed the study. J.Y., B.Q., and A.M.M. performed the experiments and collected the data. X.T. and H.D. provided reagents for the experiments. J.Y., B.Q., S.N., Z.L., and L.W. wrote and revised the manuscript. J.C.B., M. P.G., R.W., Z.L. and L.W. contributed to the interpretation of the results, conceived and supervised the project.

\section{ADDITIONAL INFORMATION}

Supplementary information accompanies this paper at https://doi.org/10.1038/ s41422-020-0315-8.

Competing interests: The authors declare no competing interests.

\section{REFERENCES}

1. Pardoll, D. M. The blockade of immune checkpoints in cancer immunotherapy. Nat. Rev. Cancer 12, 252-264 (2012).

2. Fife, B. T. \& Bluestone, J. A. Control of peripheral T-cell tolerance and autoimmunity via the CTLA-4 and PD-1 pathways. Immunol. Rev. 224, 166-182 (2008).

3. Finger, L. R. et al. The human PD-1 gene: complete CDNA, genomic organization, and developmentally regulated expression in B cell progenitors. Gene 197, 177-187 (1997).

4. Shi, L., Chen, S., Yang, L. \& Li, Y. The role of PD-1 and PD-L1 in T-cell immune suppression in patients with hematological malignancies. J. Hematol. Oncol. 6, 74 (2013).

5. Thibult, M. L. et al. PD-1 is a novel regulator of human B-cell activation. Int. Immunol. 25, 129-137 (2013).

6. Topalian, S. L., Drake, C. G. \& Pardoll, D. M. Targeting the PD-1/B7-H1(PD-L1) pathway to activate anti-tumor immunity. Curr. Opin. Immunol. 24, 207-212 (2012).

7. Dong, $\mathrm{H}$. et al. Tumor-associated $\mathrm{B} 7-\mathrm{H} 1$ promotes T-cell apoptosis: a potential mechanism of immune evasion. Nat. Med. 8, 793-800 (2002).

8. Ghebeh, H. et al. The B7-H1 (PD-L1) T lymphocyte-inhibitory molecule is expressed in breast cancer patients with infiltrating ductal carcinoma: correlation with important high-risk prognostic factors. Neoplasia 8, 190-198 (2006).

9. Villadolid, J. \& Amin, A. Immune checkpoint inhibitors in clinical practice: update on management of immune-related toxicities. Transl. Lung Cancer Res. 4, 560-575 (2015).

10. Wong, R. M. et al. Programmed death-1 blockade enhances expansion and functional capacity of human melanoma antigen-specific CTLs. Int. Immunol. 19, 1223-1234 (2007)

11. Wu, K., Kryczek, I., Chen, L. P., Zou, W. P. \& Welling, T. H. Kupffer cell suppression of CD8(+) T Cells in human hepatocellular carcinoma is mediated by B7-H1/ programmed death-1 interactions. Cancer Res. 69, 8067-8075 (2009).

12. Ahmadzadeh, M. et al. Tumor antigen-specific CD8 T cells infiltrating the tumor express high levels of PD-1 and are functionally impaired. Blood 114, 1537-1544 (2009).

13. Fourcade, J. et al. Upregulation of Tim-3 and PD-1 expression is associated with tumor antigen-specific CD8(+) T cell dysfunction in melanoma patients. J. Exp. Med. 207, 2175-2186 (2010).

14. Azuma, T. et al. B7-H1 is a ubiquitous antiapoptotic receptor on cancer cells. Blood 111, 3635-3643 (2008).

15. Orzechowski, A., Dong, H. D. Abstract 5026: B7-H1 confers tumor chemoresistance by regulating MAPK/ERK activation. Cancer Res. 74(19 Suppl):Abstract nr 5026 (2014).

16. Chang, C. H. et al. Metabolic competition in the tumor microenvironment is a driver of cancer progression. Cell 162, 1229-1241 (2015).

17. Chen, L. J. et al. B7-H1 expression associates with tumor invasion and predicts patient's survival in human esophageal cancer. Int. J. Clin. Exp. Pathol. 7, 6015-6023 (2014).

18. Mittendorf, E. A. et al. PD-L1 expression in triple-negative breast cancer. Cancer Immunol. Res. 2, 361-370 (2014).

19. Dolgin, E. Atezolizumab extends survival for breast cancer. Cancer Discov. 7, OF10 (2017).

20. Schmid, P. et al. Abstract 2986: Atezolizumab in metastatic TNBC (mTNBC): longterm clinical outcomes and biomarker analyses. Cancer Res. 77, 2986-2986 (2017).

21. Losada, A. \& Hirano, T. Dynamic molecular linkers of the genome: the first decade of SMC proteins. Genes Dev. 19, 1269-1287 (2005). 
22. Nasmyth, K. \& Haering, C. H. Cohesin: its roles and mechanisms. Annu. Rev. Genet. 43, 525-558 (2009).

23. Losada, A., Yokochi, T. \& Hirano, T. Functional contribution of Pds5 to cohesinmediated cohesion in human cells and Xenopus egg extracts. J Cell Sci. 118, 2133-2141 (2005).

24. Shintomi, K. \& Hirano, T. Releasing cohesin from chromosome arms in early mitosis: opposing actions of Wapl-Pds5 and Sgo1. Genes Dev. 23, 2224-2236 (2009).

25. Kueng, S. et al. Wapl controls the dynamic association of cohesin with chromatin. Cell 127, 955-967 (2006).

26. Nishiyama, T. et al. Sororin mediates sister chromatid cohesion by antagonizing Wapl. Cell 143, 737-749 (2010).

27. Nishiyama, T., Sykora, M. M.,'t Velda, P. J. H. I., Mechtler, K. \& Peters, J. M. Aurora B and Cdk1 mediate Wapl activation and release of acetylated cohesin from chromosomes by phosphorylating Sororin. Proc. Natl. Acad. Sci. USA 110, 13404-13409 (2013).

28. Schmitz, J., Watrin, E., Lenart, P., Mechtler, K. \& Peters, J. M. Sororin is required for stable binding of cohesin to chromatin and for sister chromatid cohesion in interphase. Curr. Biol. 17, 630-636 (2007).

29. Dorsett, D. \& Strom, L. The ancient and evolving roles of cohesin in gene expression and DNA repair. Curr. Biol. 22, R240-R250 (2012).

30. van Ruiten, M. S. \& Rowland, B. D. SMC complexes: universal DNA looping machines with distinct regulators. Trends Genet. 34, 477-487 (2018).

31. Onn, I., Heidinger-Pauli, J. M., Guacci, V., Unal, E. \& Koshland, D. E. Sister chromatid cohesion: a simple concept with a complex reality. Annu. Rev. Cell Dev. Biol. 24, 105-129 (2008).

32. Kleffel, S. et al. Melanoma cell-intrinsic PD-1 receptor functions promote tumor growth. Cell 162, 1242-1256 (2015).

33. Zhang, J. et al. Cyclin D-CDK4 kinase destabilizes PD-L1 via cullin 3-SPOP to control cancer immune surveillance. Nature 553, 91-95 (2018).

34. Li, C. W. et al. Glycosylation and stabilization of programmed death ligand-1 suppresses T-cell activity. Nat Commun. 7, 12632 (2016).

35. West, C. M. et al. (eds.) Essentials of Glycobiology. 223-238 (Cold Spring Harbor, NY, 2015).

36. Haering, C. H., Farcas, A. M., Arumugam, P., Metson, J. \& Nasmyth, K. The cohesin ring concatenates sister DNA molecules. Nature 454, 297-301 (2008).

37. Michaelis, C., Ciosk, R. \& Nasmyth, K. Cohesins: chromosomal proteins that prevent premature separation of sister chromatids. Cell 91, 35-45 (1997).

38. Guacci, V., Koshland, D. \& Strunnikov, A. A direct link between sister chromatid cohesion and chromosome condensation revealed through the analysis of MCD1 in S. cerevisiae. Cell 91, 47-57 (1997).

39. Losada, A., Hirano, M. \& Hirano, T. Identification of Xenopus SMC protein complexes required for sister chromatid cohesion. Genes Dev. 12, 1986-1997 (1998).

40. Beckouet, F. et al. Releasing activity disengages Cohesin's Smc3/Scc1 interface in a process blocked by acetylation. Mol. Cell 61, 563-574 (2016).

41. Murayama, Y. \& Uhlmann, F. DNA entry into and exit out of the cohesin ring by an interlocking gate mechanism. Cell 163, 1628-1640 (2015).

42. Gandhi, R., Gillespie, P. J. \& Hirano, T. Human Wapl is a cohesin-binding protein that promotes sister-chromatid resolution in mitotic prophase. Curr. Biol. 16, 2406-2417 (2006).
43. Ouyang, Z., Zheng, G., Tomchick, D. R., Luo, X. \& Yu, H. Structural basis and IP6 requirement for Pds5-dependent cohesin dynamics. Mol. Cell 62, 248-259 (2016).

44. Dong, $\mathrm{H}$. et al. B7-H1 determines accumulation and deletion of intrahepatic CD8 (+) T lymphocytes. Immunity 20, 327-336 (2004).

45. Wang, Y. N., Yamaguchi, H., Hsu, J. M. \& Hung, M. C. Nuclear trafficking of the epidermal growth factor receptor family membrane proteins. Oncogene 29, 3997-4006 (2010).

46. Wang, Y. N. et al. The translocon Sec61beta localized in the inner nuclear membrane transports membrane-embedded EGF receptor to the nucleus. J. Biol. Chem. 285, 38720-38729 (2010).

47. Wang, Y. N. et al. COPI-mediated retrograde trafficking from the Golgi to the ER regulates EGFR nuclear transport. Biochem. Biophys. Res. Commun. 399, 498-504 (2010)

48. Hsu, S. C. \& Hung, M. C. Characterization of a novel tripartite nuclear localization sequence in the EGFR family. J. Biol. Chem. 282, 10432-10440 (2007).

49. Wang, Y. N. et al. Membrane-bound trafficking regulates nuclear transport of integral epidermal growth factor receptor (EGFR) and ErbB-2. J. Biol. Chem. 287, 16869-16879 (2012).

50. Rondanino, C., Bousser, M. T., Monsigny, M. \& Roche, A. C. Sugar-dependent nuclear import of glycosylated proteins in living cells. Glycobiology 13, 509-519 (2003).

51. Bhosle, V. K., Rivera, J. C. \& Chemtob, S. New insights into mechanisms of nuclear translocation of G-protein coupled receptors. Small GTPases 10,254-263 (2019).

52. Zhou, L. et al. The N-terminal non-kinase-domain-mediated binding of Haspin to Pds5B Protects centromeric cohesion in mitosis. Curr Biol. 27, 992-1004 (2017).

53. Zak, K. M. et al. Structure of the complex of human programmed death 1, PD-1, and its ligand PD-L1. Structure 23, 2341-2348 (2015).

54. $\mathrm{Yu}$, J. et al. Establishing and characterizing patient-derived xenografts using prechemotherapy percutaneous biopsy and post-chemotherapy surgical samples from a prospective neoadjuvant breast cancer study. Breast Cancer Res. 19, 130 (2017).

55. Goetz, M. P. et al. Tumor sequencing and patient-derived xenografts in the neoadjuvant treatment of breast cancer. J. Natl. Cancer Inst. 109, djw306 (2017).

56. $\mathrm{Yu}$, J. et al. Regulation of serine-threonine kinase Akt Activation by NAD (+)-dependent deacetylase SIRT7. Cell Rep. 18, 1229-1240 (2017).

Open Access This article is licensed under a Creative Commons Attribution 4.0 International License, which permits use, sharing, adaptation, distribution and reproduction in any medium or format, as long as you give appropriate credit to the original author(s) and the source, provide a link to the Creative Commons license, and indicate if changes were made. The images or other third party material in this article are included in the article's Creative Commons license, unless indicated otherwise in a credit line to the material. If material is not included in the article's Creative Commons license and your intended use is not permitted by statutory regulation or exceeds the permitted use, you will need to obtain permission directly from the copyright holder. To view a copy of this license, visit http://creativecommons. org/licenses/by/4.0/.

(c) The Author(s) 2020 